\title{
Active Control of Nonlinear Suspension System Using Modified Adaptive Supertwisting Controller
}

\author{
Jagat J. Rath, ${ }^{1}$ Kalyana C. Veluvolu, ${ }^{1}$ and Michael Defoort ${ }^{2}$ \\ ${ }^{1}$ School of Electronics Engineering, College of IT Engineering, Kyungpook National University, Daegu 702701, Republic of Korea \\ ${ }^{2}$ LAMIH, CNRS UMR 8201, UVHC, 59313 Valenciennes, France \\ Correspondence should be addressed to Kalyana C. Veluvolu; veluvolu@ee.knu.ac.kr
}

Received 26 March 2015; Revised 29 June 2015; Accepted 29 June 2015

Academic Editor: Zhan Zhou

Copyright (c) 2015 Jagat J. Rath et al. This is an open access article distributed under the Creative Commons Attribution License, which permits unrestricted use, distribution, and reproduction in any medium, provided the original work is properly cited.

\begin{abstract}
The suspension system is faced with nonlinearities from the spring, damper, and external excitations from the road surface. The objective of any control action provided to the suspension is to improve ride comfort while ensuring road holding for the vehicle. In this work, a robust higher order sliding mode algorithm combining the merits of the modified supertwisting algorithm and the adaptive supertwisting algorithm has been proposed for the nonlinear active suspension system. The proposed controller is robust to linearly growing perturbations and bounded uncertainties. Simulations have been performed for different classes of road excitations and the results are presented.
\end{abstract}

\section{Introduction}

The vertical dynamics of a vehicle are governed by the behavior of its suspension and its interaction with the vehicle and tires. The suspension consists of a spring and damper unit with nonlinear properties. The suspension is connected to the vehicle chassis and the tires on either end. The interaction of the suspension unit coupled to the tire with the ground surface in ideal conditions results in minimal vibrations which are dampened out by the tire and the suspension units' damper. However, in the presence of nonlinearities and road excitations [1], it becomes imperative to provide extra control effort to damp out the effect of these perturbations. The external road excitations arise either from the varying road profiles or road perturbations in the form of ditches or bumps.

In [2] a variable gain control scheme was proposed for an active suspension system faced with road excitations and nonlinear suspension dynamics. Robust control approaches such as integral sliding mode based control and fuzzy sliding mode control for the suspension dynamics were proposed in $[3,4]$, respectively. The work in [4], which considered the presence of nonlinear suspension stiffness and damping, performed analysis considering the nonlinearities piecewise linear. Further improvisations to the suspension control under influence of actuator faults and delays were done by providing reliable fuzzy control action in [5]. The control of suspension systems employing the dynamics of hydraulic actuators has also been discussed in the literature in [6-8]. In all these approaches the focus was to provide efficient control action to the suspension system ensuring ride quality and road holding at the same time.

Sliding mode [9-11] control has been established over time as a robust technique against perturbations and uncertainties. The works $[9,10]$ focused on developing sliding mode based observers for nonlinear systems faced with uncertainties and nonlinearities. The applications of sliding mode technique in combination with other high gain techniques were further explored in [11]. Sliding mode has been effectively employed to generate control for active suspension systems as discussed in [3, 8]. In [8] an output feedback control scheme based on first order sliding mode for an active suspension system faced with actuator nonlinearities and road disturbances was proposed. Similarly in [3] an integral sliding mode control was proposed for the linear model of an active suspension system. In [12] a first order sliding mode controller was proposed for the linear active suspension system presenting a comparative analysis between passive and actively controlled suspension system. However these approaches employed first order sliding mode techniques and hence were affected by chattering problems $[13,14]$. 
The emergence of higher order sliding mode (HOSM) and particularly the supertwisting algorithm (STA) for systems with relative degree one has led to the reduction in chattering while providing the necessary control. Applications of higher order sliding mode control [15] in fields of unknown input estimations [16], vehicle control [17], and fuel cell systems [18] amongst others have been successfully explored. By improving on the disturbance rejections attributes of the STA, in [19-21] a generalized STA was proposed to ensure that linearly growing perturbations are also rejected by the control action. In [22] an adaptive STA based technique was proposed which focused on minimizing the chattering effect. Similarly in $[23,24]$ an adaptive STA based control action for an electropneumatic actuator was proposed. In this work the proposed bounded gains of the STA adapt to reject the linear perturbations and force the convergence of sliding mode in finite time. Higher order sliding mode based controllers for active suspension systems were proposed in $[25,26]$. In both these works the second order sliding mode controllers based on STA were employed for the linear suspension system. The works in $[5,12,25,26]$ did not consider the nonlinear dynamics of the active suspension system. Similarly the works in $[2,3,12,25,26]$ did not consider the presence of road roughness profile.

To address these issues, in this paper an adaptive higher order sliding mode controller is proposed for the nonlinear active suspension system. The nonlinear model of the active suspension systems for a quarter car is first developed by integrating the suspension nonlinearities due to damper and spring and the road excitations as disturbance inputs to the model. For analysis, the road roughness values based on power spectral density (PSD) values as proposed by International Organization for Standardization (ISO) [1] have been employed. Based on the roughness index different classes or road profiles are generated which serve as the external excitations to the suspension system. The influence of tire damping generally neglected in other works has also been considered in this work. For the nonlinear suspension system, a control based on modified adaptive STA is proposed to reduce the sprung mass acceleration and hence improve ride comfort. The proposed control also ensures that road holding condition is ensured such that the vehicle does not leave the ground and lead to instability. Analysis of the control performance for different classes of road excitations was performed and simulation results have been provided.

\section{Problem Formulation}

The dynamics of the suspension in the presence of spring and damper nonlinearities and road excitations can be given as $[1,6]$

$$
\begin{aligned}
M_{s} \ddot{z}_{s}= & -K_{s} z_{\mathrm{su}}-B_{s} \dot{z}_{\mathrm{su}}-\varphi+U_{s}, \\
M_{u} \ddot{z}_{u}= & K_{s} z_{\mathrm{su}}+B_{s} z_{\mathrm{su}}-K_{t}\left(z_{u}-\xi\right)-B_{t}\left(\dot{z}_{u}-\dot{\xi}\right)+\varphi \\
& -U_{s},
\end{aligned}
$$

where $M_{s}$ represents the chassis/sprung mass, $M_{u}$ is the tire/unsprung mass, $K_{s}$ and $B_{s}$ are the spring stiffness and

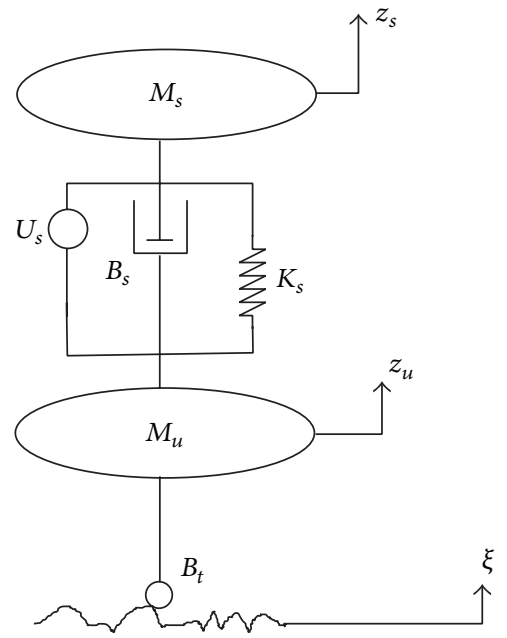

FIGURE 1: The active suspension system.

damping, respectively, $K_{t}$ and $B_{t}$ represent the tire stiffness and damping, respectively, $U_{s}$ represents the active control input to be designed later, and $\xi$ represents the external road excitation. The motions of the suspension system are represented by the sprung mass acceleration $\ddot{z}_{s}$ and the unsprung mass acceleration $\ddot{z}_{u}$. Further, $z_{\text {su }}$ represents the suspension stroke/displacement and $z_{u}$ is the unsprung mass displacement. The nonlinearity $\varphi$ in the suspension dynamics is given as [1]

$$
\varphi=K_{\mathrm{nl}} z_{\mathrm{su}}^{3}+B_{\mathrm{nl}} \sqrt{\left|\dot{z}_{\mathrm{su}}\right|} \operatorname{sign}\left(\dot{z}_{\mathrm{su}}\right),
$$

with $K_{\mathrm{nl}}$ and $B_{\mathrm{nl}}$ representing the nonlinear percentages of the spring stiffness and damping, respectively. For the suspension system modeled in (1) the objective of the active control input $U_{s}$ in general varies with the specifications of applicability. The schematic representation of the suspension system is shown in Figure 1. The control input can be provided to improve the ride comfort or to ensure proper road holding. It may also be provided to minimize the rattle space and ensure the vehicle static weight is well supported. Ride comfort enhancement can be achieved by the reduction in the sprung mass acceleration while road holding can be ensured by ensuring a reduction in tire deflection, $z_{\xi}=\left(z_{u}-\xi\right)$. Similarly the rattle space can be limited by fixing the maximum possible suspension stroke with a suitable control effort. However it can be deduced from (1) that the control effort cannot achieve these objectives at the same time. Hence, the problem is formulated as providing control input for an underactuated suspension system with objective of reduction in sprung mass acceleration only. The control action provided abides by the following constraints:

(1) Limiting Suspension Stroke [5]. The provided suspension control while achieving the increase in ride comfort must limit the suspension stroke to a mechanical constraint based on the design of the suspension. Hence, we have

$$
\left|z_{\text {su }}\right| \leq|Z|_{\max } .
$$




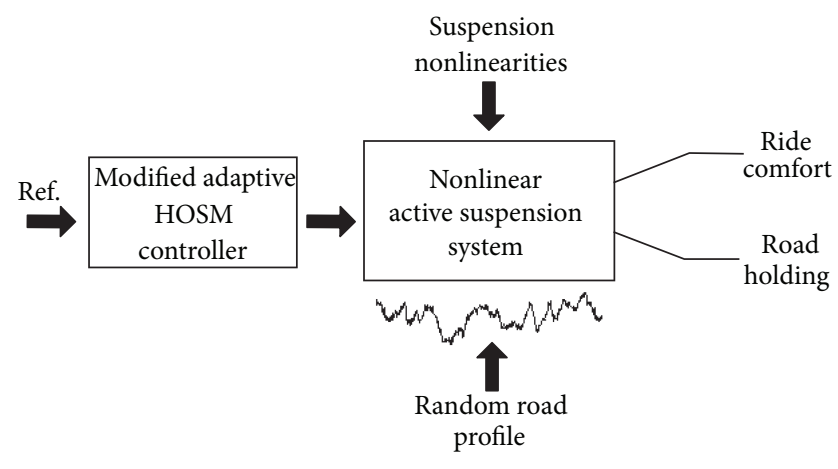

FIGURE 2: The block representation of the proposed control.

(2) Road Holding [5]. It is intended to ensure that the control does not cause the vehicle to leave the ground and cause instability. Hence, we have

$$
K_{t} z_{\xi} \leq\left(M_{s}+M_{u}\right) g .
$$

This ensures that the dynamic tire loading is less than the static vertical load and the tire does not leave the ground vertically.

Under the above control constraints, we propose an active control action based on the adaptive modified supertwisting HOSM algorithm to achieve the control objective of improvement in ride comfort by reduction in sprung mass acceleration. The block diagram representation of the proposed scheme has been shown in Figure 2.

\section{HOSM Controller Design}

In this section we discuss the design of the proposed adaptive modified STA based controller aimed at reducing the sprung mass acceleration. For the design of the controller, we can formulate the following state space model with the states as

$$
x=\left[\begin{array}{llll}
x_{1} & x_{2} & x_{3} & x_{4}
\end{array}\right]^{T}=\left[\begin{array}{llll}
z_{\mathrm{su}} & \dot{z}_{s} & z_{\xi} & \dot{z}_{u}
\end{array}\right]^{T}
$$

$\dot{x}$

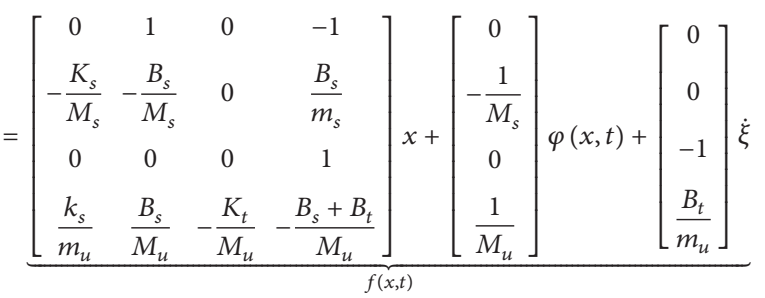

$$
+\underbrace{\left[\begin{array}{c}
0 \\
\frac{1}{M_{s}} \\
0 \\
-\frac{1}{m_{u}}
\end{array}\right]}_{g(x, t)}
$$

The above dynamics of the suspension can be expressed as

$$
\dot{x}=f(x, t)+g(x, t) u .
$$

For the above system, the following sliding surface is selected:

$$
s=\dot{z}_{\text {su }}
$$

As system (5)-(7) has a relative degree one for $s$ with respect to the input, $U_{s}$, the dynamics of the sliding surface can be thus obtained as

$$
\dot{s}=\left(\ddot{z}_{s}-\ddot{z}_{u}\right)=\alpha(x, t)+M \nu(s),
$$

where $M=\left(M_{s}+M_{u}\right) / M_{s} M_{u}$ and $\nu(s)$ is the robust sliding mode control input designed based on the adaptive modified STA given as

$$
\begin{aligned}
& v=-\kappa_{1} \phi_{1}(s(t))+\omega, \\
& \dot{w}=-\kappa_{2} \phi_{2}(s(t)),
\end{aligned}
$$

with $\phi_{1}(s(t))=s+\kappa_{3}|s|^{1 / 2} \operatorname{sign}(s)$ and $\phi_{2}(s(t))=s+$ $\left(\kappa_{3}^{2} / 2\right) \operatorname{sign}(s)+(3 / 2) \kappa_{3}|s|^{1 / 2} \operatorname{sign}(s)$. The adaptive gains are given as [24]

$$
\begin{aligned}
& \dot{\kappa}_{1}= \begin{cases}\omega_{1} \sqrt{\frac{\mu_{1}}{2}} \operatorname{sign}(|s|-\delta), & \text { if } \kappa_{1}>\delta_{m} \\
\zeta & \text { if } \kappa_{1} \leq \delta_{m},\end{cases} \\
& \kappa_{2}=2 \epsilon \kappa_{1},
\end{aligned}
$$

with $\kappa_{3}, \mu_{1}, \delta, \zeta, \epsilon$, and $\omega_{1}$ being positive constants. The conditions $\zeta_{1} \geq \delta, \zeta_{2}>0$ hold where $\delta_{m}$ is an arbitrary small constant. With this control law, the perturbation term $\alpha(x, t)$ for system (8) is now given as

$$
\begin{aligned}
\alpha(x, t)= & M\left(-K_{s} z_{\text {su }}-B_{s} \dot{z}_{\text {su }}-\varphi(x, t)\right)+\frac{K_{t}}{M_{u}} z_{\xi} \\
& +\frac{B_{t}}{M_{u}} \dot{z}_{\xi}=\alpha_{1}(x, t)+\alpha_{2}(x, t) .
\end{aligned}
$$

The perturbation terms are then given as

$$
\begin{aligned}
\alpha_{1}(x, t)= & M B_{s} \dot{z}_{\mathrm{su}}, \\
\alpha_{2}(x, t)= & M\left(-K_{s} z_{\mathrm{su}}-2 B_{s} \dot{z}_{\mathrm{su}}-\varphi(x, t)\right)+\frac{K_{t}}{M_{u}} z_{\xi} \\
& +\frac{B_{t}}{M_{u}} \dot{z}_{\xi} .
\end{aligned}
$$

The term $\alpha_{2}$ consists of nonlinear terms and disturbances. The term $\varphi(x, t)$ is Lipschitz bounded whose Lipschitz constant can be easily computed as discussed in [1]. The quantity $\xi$ refers to the road excitation that serves as a disturbance input for system (5). It can be assumed that this entity is bounded although its bound is not known a priori. The other terms in the disturbance $\alpha_{2}$ are functions of the system which are bounded for all practical operating conditions. Hence the boundedness of the disturbance terms in (14) can be established as

$$
\begin{aligned}
& \left|\alpha_{1}(x, t)\right| \leq \rho_{1}|s|, \\
& \left|\dot{\alpha}_{2}(x, t)\right| \leq \rho_{2} .
\end{aligned}
$$


The disturbance term $\alpha_{2}(x, t)$ consists of the suspension system dynamics and the disturbance input $\xi$, which is bounded but whose exact values are not known. Hence the value of the bound $\rho_{2}$ cannot be determined a priori.

Theorem 1. With condition (15) satisfied, controller (9)-(10) will ensure that the sliding surface (s) converges to a practically stable equilibrium in finite time.

Proof. To establish the convergence, the sliding dynamics (8) can be rewritten along with the control input as

$$
\begin{aligned}
& \dot{s}_{1}=s_{2}-\kappa_{1} \phi_{1}\left(s_{1}\right)+\alpha_{1}(x, t), \\
& \dot{s}_{2}=-\kappa_{2} \phi_{2}\left(s_{1}\right)+\dot{\alpha}_{2}(x, t),
\end{aligned}
$$

with $s_{1}=s$ and $s_{2}=\alpha_{2}(x, t)+\beta_{1}(x, t) \omega$. One could notice that gain $\kappa_{3}$ is used to withstand linearly growing perturbations. Since $\left|\phi_{2}(s)\right| \geq \kappa_{3}^{2} / 2$ one can obtain

$$
\left|\dot{\alpha}_{2}\right|<\frac{2 \rho_{2}}{\kappa_{3}^{2}} \phi_{2}(s) .
$$

We consider a new state vector as [19]

$$
\xi=\left[\begin{array}{l}
\xi_{1} \\
\xi_{2}
\end{array}\right]=\left[\begin{array}{c}
\phi_{1}\left(s_{1}\right) \\
s_{2}
\end{array}\right] .
$$

Thus, system (17) can be rewritten as

$$
\begin{gathered}
\dot{\xi}=\left[\begin{array}{c}
\left(1+\frac{\kappa_{3}}{2}\left|s_{1}\right|^{-1 / 2}\right)\left(s_{2}-\kappa_{1} \phi_{1}\left(s_{1}\right)+\alpha_{1}\right) \\
-\kappa_{2} \phi_{2}\left(s_{1}\right)+\dot{\alpha}_{2}
\end{array}\right] \\
=\left(1+\frac{\kappa_{3}}{2}\left|s_{1}\right|^{-1 / 2}\right)\left(A_{0} \xi+\left[\begin{array}{c}
\alpha_{1} \\
0
\end{array}\right]\right)+\left[\begin{array}{c}
0 \\
\dot{\alpha}_{2}
\end{array}\right],
\end{gathered}
$$

where $A_{0}$ is a Hurwitz matrix selected as

$$
A_{0}=\left[\begin{array}{ll}
-\kappa_{1} & 1 \\
-\kappa_{2} & 0
\end{array}\right] \text {. }
$$

To perform the stability analysis of (17), the following Lyapunov candidate function is selected:

$$
V(\xi, \kappa)=V_{\xi}+\frac{1}{2 \mu_{1}}\left(\kappa_{1}-\kappa_{1}^{*}\right)^{2}+\frac{1}{2 \mu_{2}}\left(\kappa_{2}-\kappa_{2}^{*}\right)^{2},
$$

where $\kappa_{1}^{*}>0$ and $\kappa_{2}^{*}>0$ are some constants. The function $V_{\xi}$ is given as [19]

$$
V_{\xi}=\xi^{T} P \xi
$$

with $P=P^{T}=\left[\begin{array}{cc}\lambda+4 \epsilon^{2} & -2 \epsilon \\ -2 \epsilon & 1\end{array}\right], \lambda>0$ and $\epsilon>0$. It is worth noting that matrix $P$ is positive definite if $\lambda$ and $\epsilon$ are any real number. The time derivative of $V_{\xi}$ can be obtained as

$$
\begin{aligned}
\dot{V}_{\xi}= & \left(1+\frac{\kappa_{3}}{2}\left|s_{1}\right|^{-1 / 2}\right) \\
& \cdot\left(\xi^{T}\left(A_{0}^{T} P+P A_{0}\right) \xi+2 \xi^{T} P\left[\begin{array}{c}
\alpha_{1} \\
0
\end{array}\right]\right) \\
& +2 \xi^{T} P\left[\begin{array}{c}
0 \\
\dot{\alpha}_{2}
\end{array}\right] .
\end{aligned}
$$

From (18), one can note that

$$
\begin{aligned}
\left|2 \xi^{T} P\left[\begin{array}{c}
0 \\
\dot{\alpha}_{2}
\end{array}\right]\right| & \leq\left|2 \xi^{T} P\left[\begin{array}{c}
0 \\
\frac{2 \rho_{2}}{\kappa_{3}^{2}} \phi_{2}
\end{array}\right]\right| \\
& =\left|\left(1+\frac{\kappa_{3}}{2}\left|s_{1}\right|^{-1 / 2}\right) 2 \xi^{T} P\left[\begin{array}{c}
0 \\
\frac{2 \rho_{2}}{\kappa_{3}^{2}} \xi_{1}
\end{array}\right]\right| .
\end{aligned}
$$

Denoting $2 \rho_{2} / \kappa_{3}^{2}=\rho_{3}$, where $\rho_{3}$ is unknown, we thus obtain

$$
\begin{aligned}
\dot{V}_{\xi} \leq & \left(1+\frac{\kappa_{3}}{2}\left|s_{1}\right|^{-1 / 2}\right) \\
& \cdot\left(\xi^{T}\left(A_{0}^{T} P+P A_{0}\right) \xi+2 \xi^{T} P\left[\begin{array}{c}
\alpha_{1} \\
0
\end{array}\right]\right) \\
& +\left(1+\frac{\kappa_{3}}{2}\left|s_{1}\right|^{-1 / 2}\right) 2 \xi^{T} P\left[\begin{array}{c}
0 \\
\rho_{3} \xi_{1}
\end{array}\right] .
\end{aligned}
$$

Using bounds on the perturbation (15), we can obtain

$$
\begin{aligned}
\dot{V}_{\xi} \leq & \left(1+\frac{\kappa_{3}}{2}\left|s_{1}\right|^{-1 / 2}\right) \\
& \cdot\left(\xi^{T}\left(A_{0}^{T} P+P A_{0}\right) \xi+2 \xi^{T} P\left[\begin{array}{c}
\rho_{1} \xi_{1} \\
0
\end{array}\right]\right) \\
& +\left(1+\frac{\kappa_{3}}{2}\left|s_{1}\right|^{-1 / 2}\right) 2 \xi^{T} P\left[\begin{array}{c}
0 \\
\rho_{3} \xi_{1}
\end{array}\right] .
\end{aligned}
$$

Furthermore, one can obtain, similar to [19],

$$
\dot{V}_{\xi} \leq-\left(1+\frac{\kappa_{3}}{2}\left|s_{1}\right|^{-1 / 2}\right) \xi^{T} Q \xi
$$

with

$$
\begin{aligned}
Q & =\left[\begin{array}{ll}
Q_{1} & Q_{2} \\
Q_{2} & Q_{3}
\end{array}\right], \\
Q_{1} & =2\left(\kappa_{1}-\rho_{1}\right)\left(\lambda+4 \epsilon^{2}\right)-4 \epsilon\left(\kappa_{2}-\rho_{3}\right), \\
Q_{2} & =\left(\kappa_{2}-2 \epsilon \kappa_{1}\right)+2 \epsilon \rho_{1}+\rho_{3}-\left(\lambda+4 \epsilon^{2}\right), \\
Q_{3} & =4 \epsilon .
\end{aligned}
$$

In order to guarantee the positive definiteness of matrix $Q$, we can choose

$$
\kappa_{2}=2 \epsilon \kappa_{1} .
$$

The matrix $Q$ is positive definite if

$$
\begin{aligned}
\kappa_{1}> & \frac{\rho_{1} \lambda \epsilon+4 \rho_{1} \epsilon^{3}-2 \epsilon^{2} \rho_{3}}{\lambda \epsilon} \\
& +\frac{\left(2 \epsilon \rho_{1}+\rho_{3}-\left(\lambda+4 \epsilon^{2}\right)\right)^{2}}{8 \lambda \epsilon} .
\end{aligned}
$$


From (19), it can be deduced that

$$
\|\xi\|^{2}=\xi_{1}^{2}+\xi_{2}^{2} \geq \kappa_{3}^{2}\left|s_{1}\right| .
$$

It can be further shown that

$$
\dot{V}_{\xi}=-r_{1} V_{\xi}^{1 / 2}-r_{2} V_{\xi}
$$

with

$$
\begin{aligned}
& r_{1}=-\gamma \frac{\lambda_{\text {min }}(Q)}{\lambda_{\text {max }}^{1 / 2}(P)} \frac{\kappa_{3}^{2}}{2}, \\
& r_{2}=\gamma \frac{\lambda_{\text {min }}(Q)}{\lambda_{\text {max }}(P)}
\end{aligned}
$$

with $1>\gamma>0$.

Considering $\gamma_{\kappa_{1}}=\kappa_{1}-\kappa_{1}^{*}$ and $\gamma_{\kappa_{2}}=\kappa_{2}-\kappa_{2}^{*}$, the time derivative of the Lyapunov candidate function $V\left(\xi_{1}, \xi_{2}, \kappa_{1}, \kappa_{2}\right)$ can thus be written as

$$
\begin{aligned}
\dot{V}(\xi, \kappa) \leq & -r_{1} V_{\xi}^{1 / 2}-r_{2} V_{\xi}+\frac{1}{\mu_{1}} \gamma_{\kappa_{1}} \dot{\kappa}_{1}+\frac{1}{\mu_{2}} \gamma_{\kappa_{2}} \dot{\kappa}_{2} \\
\leq & -r_{1} V_{\xi}^{1 / 2}-r_{2} V_{\xi}-\frac{\omega_{1}}{\sqrt{2 \mu_{1}}}\left|\gamma_{\kappa_{1}}\right|-\frac{\omega_{2}}{\sqrt{2 \mu_{2}}}\left|\gamma_{\kappa_{2}}\right| \\
& +\frac{1}{\mu_{1}} \gamma_{\kappa_{1}} \dot{\kappa}_{1}+\frac{1}{\mu_{2}} \gamma_{\kappa_{2}} \dot{\kappa}_{2}+\frac{\omega_{1}}{\sqrt{2 \mu_{1}}}\left|\gamma_{\kappa_{1}}\right| \\
& +\frac{\omega_{2}}{\sqrt{2 \mu_{2}}}\left|\gamma_{\kappa_{2}}\right| .
\end{aligned}
$$

Employing the inequality

$$
\left(x^{2}+y^{2}+z^{2}\right)^{1 / 2} \leq|x|+|y|+|z|,
$$

one could obtain

$$
\begin{aligned}
& -r_{1} V_{\xi}^{1 / 2}-\frac{\omega_{1}}{\sqrt{2 \mu_{1}}}\left|\gamma_{\kappa_{1}}\right|-\frac{\omega_{2}}{\sqrt{2 \mu_{2}}}\left|\gamma_{\kappa_{2}}\right| \\
& \leq-\gamma_{1} \sqrt{V\left(\xi_{1}, \xi_{2}, \kappa_{1}, \kappa_{2}\right)}
\end{aligned}
$$

where $\gamma_{1}=\min \left(r_{1}, \omega_{1}, \omega_{2}\right)$. Thus, the following can be written:

$$
\begin{aligned}
\dot{V}(\xi, \kappa) \leq & -\gamma_{1} \sqrt{V(\xi, \kappa)}-r_{2} V_{\xi}+\frac{1}{\mu_{1}} \gamma_{\kappa_{1}} \dot{\kappa}_{1}+\frac{1}{\mu_{2}} \gamma_{\kappa_{2}} \dot{\kappa}_{2} \\
& +\frac{\omega_{1}}{\sqrt{2 \mu_{1}}}\left|\gamma_{\kappa_{1}}\right|+\frac{\omega_{2}}{\sqrt{2 \mu_{2}}}\left|\gamma_{\kappa_{2}}\right| .
\end{aligned}
$$

Thus the following can now be written:

$$
\dot{V}(\xi, \kappa) \leq-\gamma_{1} \sqrt{V(\xi, \kappa)}-r_{2} V_{\xi}+\Delta
$$

with $\Delta=-\left|\gamma_{\kappa_{1}}\right|\left(\dot{\kappa}_{1} / \mu_{1}-\omega_{1} / \sqrt{2 \mu_{1}}\right)-\left|\gamma_{\kappa_{2}}\right|\left(\dot{\kappa}_{2} / \mu_{2}-\omega_{2} / \sqrt{2 \mu_{2}}\right)$. Now, considering that $\left|s_{1}\right|>\delta$ and $\kappa_{1}>\delta_{m} \forall t>0$, we can obtain from (11)

$$
\begin{aligned}
& \dot{\kappa}_{1}=\omega_{1} \sqrt{\frac{\mu_{1}}{2}} \\
& \Delta=-\left|\gamma_{\kappa_{2}}\right|\left(\dot{\kappa}_{2}-\frac{\omega_{2}}{\sqrt{2 \mu_{2}}}\right) .
\end{aligned}
$$

Taking $\epsilon=\left(\omega_{2} / 2\right) \sqrt{\mu_{2} / \mu_{1}}$, we can obtain, with $\Delta=0$,

$$
\dot{V}(\xi, \kappa) \leq-\gamma_{1} \sqrt{V(\xi, \kappa)}-r_{2} V_{\xi}
$$

For the finite time convergence it is necessary that $\kappa_{1}$ satisfies inequality (31). It implies that $\kappa_{1}$ increases in accordance with (40) until (31) is met which guarantees the positive definiteness of matrix Q and validity of (42) in finite time to the domain $|s| \leq \delta$.

In the condition when $|s|<\delta$, the adaptive gain $\kappa_{1}$ can be obtained as

$$
\dot{\kappa}_{1}= \begin{cases}-\left(\omega_{1} \sqrt{\frac{\mu_{1}}{2}}\right), & \text { if } \kappa_{1}>\delta_{m} \\ \zeta & \text { if } \kappa_{1} \leq \delta_{m}\end{cases}
$$

and the term $\Delta$ is given as

$\Delta$

$$
= \begin{cases}2\left(\left|\kappa_{1}-\kappa_{1}^{*}\right| \sqrt{\frac{1}{2 \mu_{1}}}\right), & \text { if } \kappa_{1}>\delta_{m} \\ -\left|\delta_{m}-\kappa_{1}^{*}+\zeta \cdot t\right|\left(\frac{\zeta}{\mu_{1}}-\frac{\omega_{1}}{\sqrt{2 \mu_{1}}}\right) & \text { if } \kappa_{1} \leq \delta_{m} .\end{cases}
$$

It can be deduced that second equation in (44) is valid for a finite duration. As $\kappa_{1}$ soon becomes less than or equal to $\delta_{m}$, its value starts increasing such that $\kappa_{1}=\delta_{m}+\zeta \cdot t$. Then the first equation in (44) is valid. The derivative of the Lyapunov function (39) becomes sign indefinite and $|s|$ may become larger than $\delta$ due to the decrease in the adaptive gains. During the adaptation process the sliding variable reaches the domain $|s|<\delta$ in finite time and then may leave the domain in finite time. It is however guaranteed that it always stays in a larger domain $|s| \leq \zeta_{1}, \zeta_{1}>\delta$ in the real sliding mode. It can be shown, similar to [24], that the real sliding mode is defined in the domain

$$
W=\left\{s_{1}, \dot{s}_{1}:\left|s_{1}\right| \leq \zeta_{2}, \zeta_{1}>\delta\right\} .
$$

Remark 2. The adaptive gains $\kappa_{1}$ and $\kappa_{2}$ are bounded. In the domain $\delta<\left|s_{1}\right| \leq \zeta_{1}$, a solution to (11) can be obtained as

$$
\kappa_{1}=\kappa_{1}(0)+\omega_{1} \sqrt{\frac{\mu_{1}}{2}} \cdot t, \quad 0 \leq t \leq t_{1},
$$

where $t_{1}$ is finite time. Thus the gain $\kappa_{1}$ is bounded, and since $\kappa_{2}$ is a function of $\kappa_{1}$ hence it is also bounded.

\section{Results and Discussion}

To validate the proposed control scheme, the quarter and active suspension parameters have been considered as $M_{s}=$ $342.5 \mathrm{~kg}, M_{u}=40 \mathrm{~kg}, K_{s}=20000 \mathrm{~N} / \mathrm{m}, B_{s}=1000 \mathrm{Ns} / \mathrm{m}$, $K_{t}=268000 \mathrm{~N} / \mathrm{m}$, and $B_{t}=14.6 \mathrm{Ns} / \mathrm{m}$. The parameters for the design of adaptive modified STA controller were selected as $K_{3}=310, \omega_{1}=150, \zeta=4.5, \delta_{m}=4.5, \delta=2, \mu_{1}=2$, and 


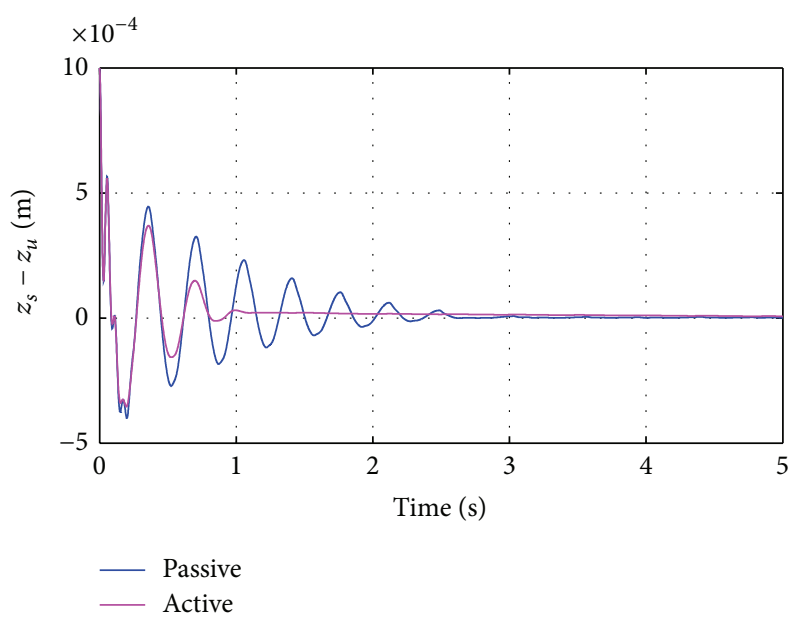

(a)

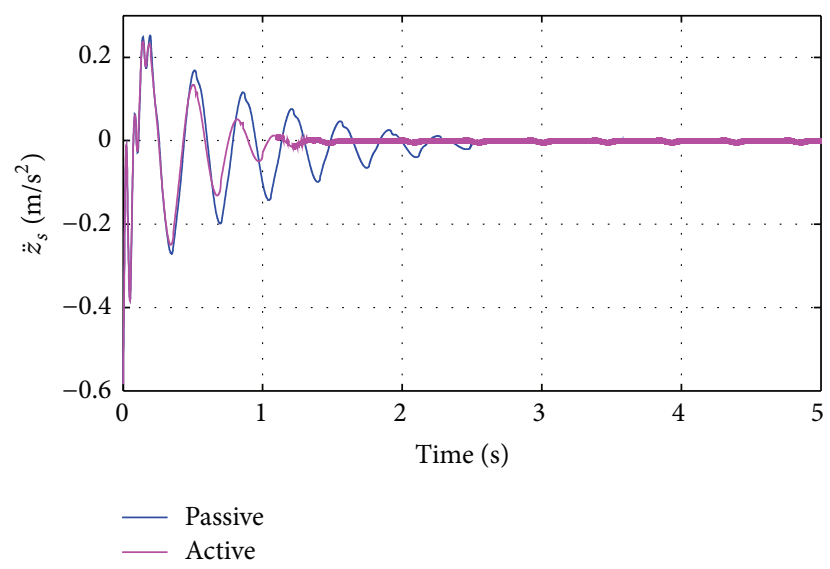

(c)

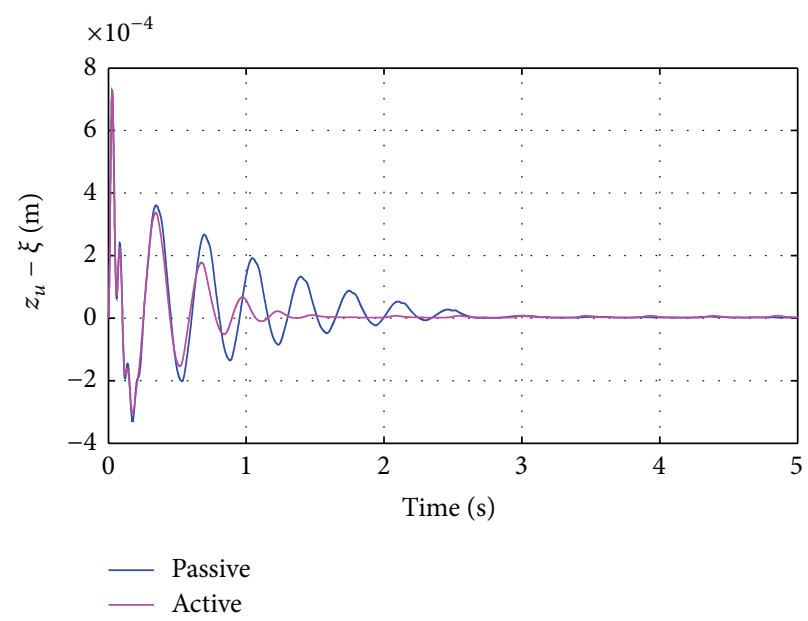

(b)

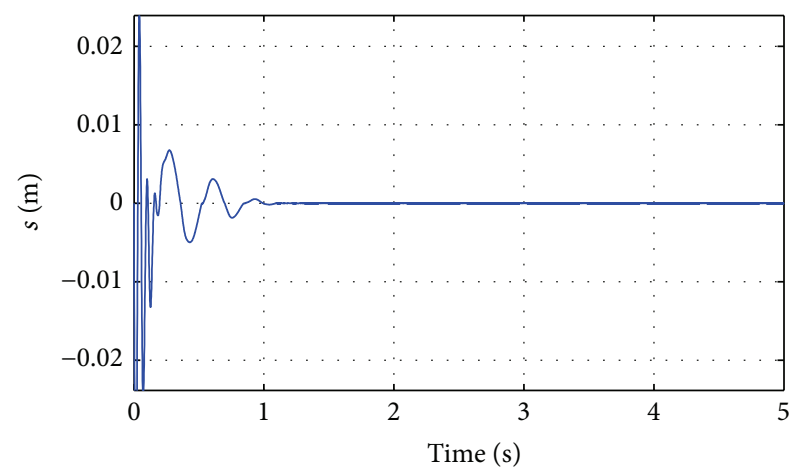

Figure 3: No road profile: (a) suspension deflection, (b) tire deflection, (c) sprung mass acceleration, and (d) sliding surface.

$\epsilon=0.0021$ such that (30)-(31) are complied with. These gains have been selected after repeated trials such that developed controller is robust for different classes of road profiles and ensures high performance. Two cases considering the absence and presence of external road excitation were considered to show the effectiveness of the proposed scheme.

4.1. Case A: No Road Excitation. The analysis was initially focused on the dynamics of the suspension system in the absence of any road profile (i.e., $\xi=0$ ). In such a scenario the suspension deflection $\left(z_{\mathrm{su}}\right)$, tire deflection $\left(z_{\xi}\right)$, and sprung mass acceleration $\left(\ddot{z}_{s}\right)$ were analyzed. In Figures 3(a)-3(c), the variations in suspension deflection, tire deflection, and the sprung mass accelerations have been shown, respectively. A comparison between the above suspension measurements in the passive control (no control input) mode and the active control (control input based on adaptive modified STA) has been also shown. During the passive control, the RMS (root mean square) value of the sprung mass acceleration was obtained as $0.0724 \mathrm{~m} / \mathrm{s}^{2}$. In comparison the RMS value of the sprung mass acceleration was obtained as $0.0588 \mathrm{~m} / \mathrm{s}^{2}$. It has been further shown in Figure 3(d) that the sliding surface is attained and converges to the origin.

4.2. Case B: External Road Profile. In this case different classes of road profiles were considered with the road conditions varying from good to very poor roads. The vehicle was considered to be traveling at a constant longitudinal speed of $37 \mathrm{~km} / \mathrm{hr}$. The road excitation was modeled as a random process with a ground displacement PSD given as

$$
\dot{\xi}(t)=-2 \pi n_{0} v \xi(t)+2 \pi \sqrt{\sigma_{0} v} w_{0},
$$

where $v$ is the vehicle longitudinal velocity, $\sigma_{0}$ is the road roughness coefficient, $n_{0}$ is the reference space frequency, and $w_{0}$ is the Gaussian white noise. Under such driving conditions varying degrees of roughness $\left(\sigma_{0}\right)$ were considered to replicate different classes of road profile. Based on the degree of roughness [1], the road classes were determined as $\operatorname{good}\left(\sigma_{0}=16 \times 10^{-6}\right)$, average $\left(\sigma_{0}=64 \times 10^{-6}\right)$, poor $\left(\sigma_{0}=256 \times 10^{-6}\right)$, and very poor $\left(\sigma_{0}=1024 \times 10^{-6}\right)$. The PSD of different classes of roads profile has been shown in Figure 4. It can be clearly seen that with increase in road roughness the PSD of the excitations increases. 


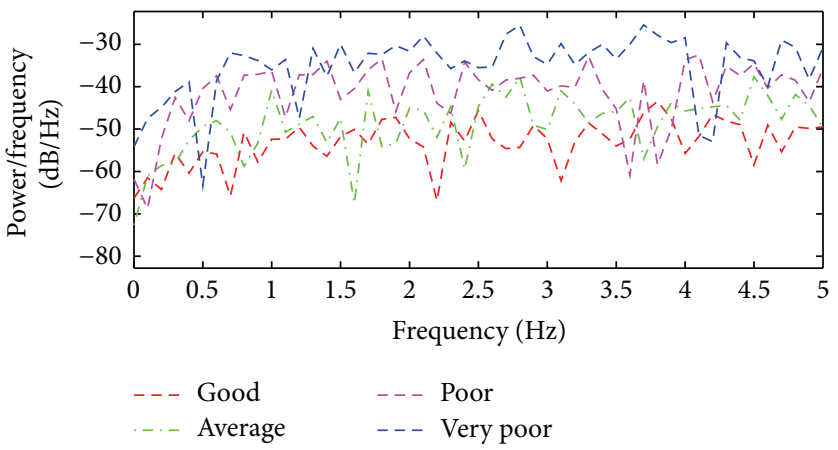

FIGURE 4: PSD for different road classes excitation.

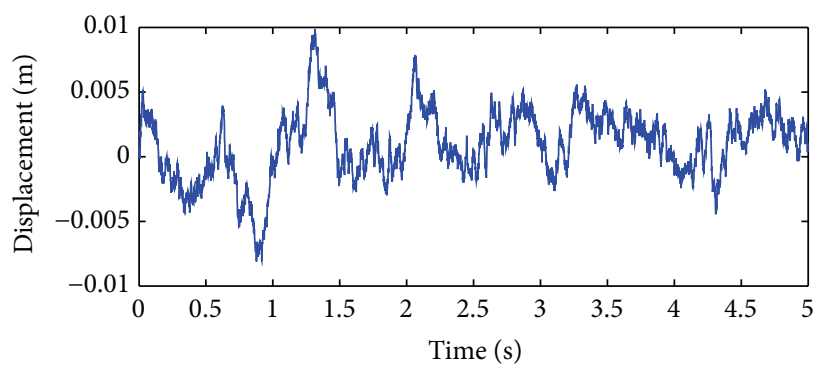

(a)

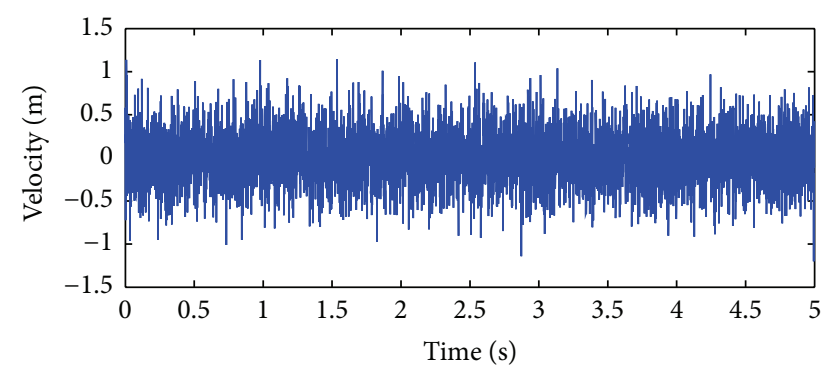

(b)

FIGURE 5: (a) Road profile for poor road. (b) Rate of road profile for poor road.

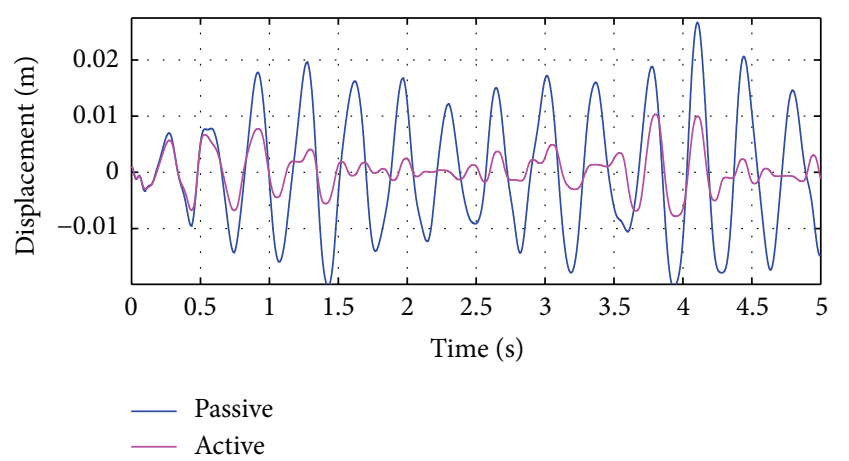

Figure 6: The suspension deflection, $z_{\text {su }}$.

For different classes of road profile discussed earlier, we selected the poor road as an external excitation and the simulation was carried out. For poor class road, the road excitation $\xi$ and the rate of road excitation have been shown in Figure 5.

To meet the control objective of increase in ride comfort by reducing the sprung mass acceleration, first it has been ensured that the control constraints discussed in (3)-(4) have been met. Thus under the effect of the active control action proposed in this work the reduction in suspension deflection magnitude in comparison to the passive case has been shown in Figure 6. The reduction in suspension deflection caters to the control constraint discussed earlier in (3) and is less than $0.01 \mathrm{~m}$.
While ensuring that the suspension stroke is limited, the effective tire load has been shown in Figure 7(a). It can be seen that the dynamic load is less than the static load which meets the control constraint discussed in (4). With the control constraints met, the control effort that ensures the control objective is met is shown in Figure 7(b). In Figure 8 the adaptive gains $K_{1}$ and $K_{2}$ of the proposed adaptive control scheme in (10) have been shown.

It can be seen that the gains change based on the perturbation and are not unbounded. Under the effect of this control action, the sliding surface which represents the rate of suspension stroke movement has been shown in Figure 9. It can be seen that the sliding surface has converged to a finite bound, thus ensuring practical stability.

With the convergence of the sliding surface, the control objective of reduction in sprung mass acceleration is met and shown in Figure 10. It can be seen that in comparison to the passive suspension system there is a reduction in magnitude of sprung mass acceleration. During the passive control, the RMS value of the sprung mass acceleration was obtained as $0.0724 \mathrm{~m} / \mathrm{s}^{2}$. In comparison the RMS value of the sprung mass acceleration was obtained as $0.0588 \mathrm{~m} / \mathrm{s}^{2}$ during active control. In Figure 11 the PSD of the sprung mass acceleration has been shown. It can be seen that under the effect of control action the performance of active controlled suspension is better in the frequency range of $1 \mathrm{~Hz}$ to $5 \mathrm{~Hz}$ which corresponds to the ride comfort frequency range. Thus it can be concluded that the proposed control action achieves the desired control objective by enhancing ride comfort. 


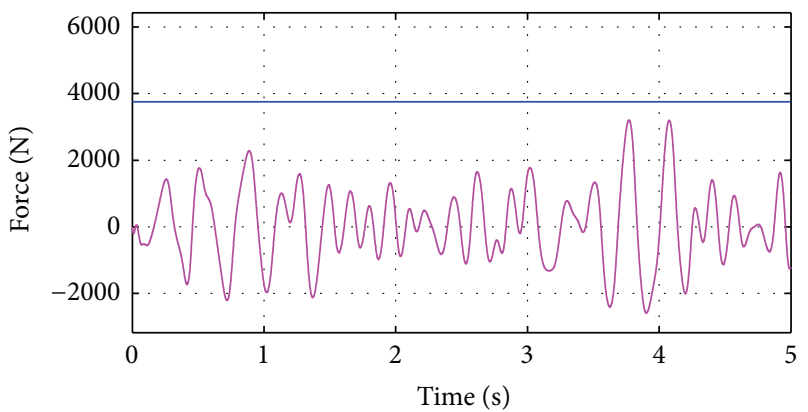

- Dynamic load

— Static load

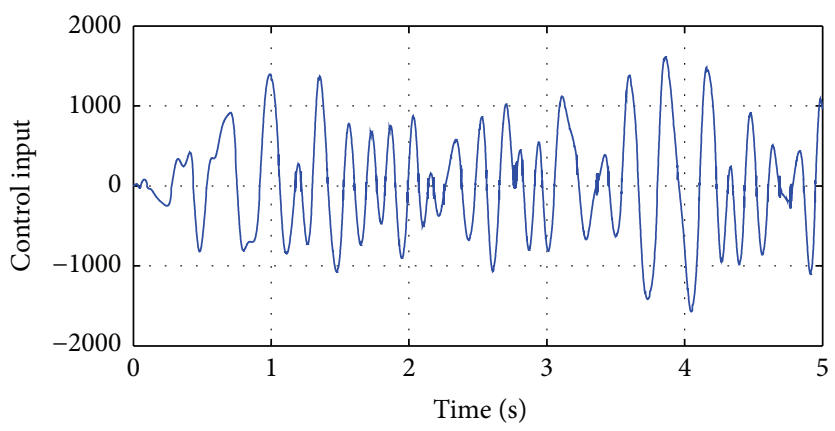

(a)

Figure 7: (a) Effective tire loading. (b) Control input provided.

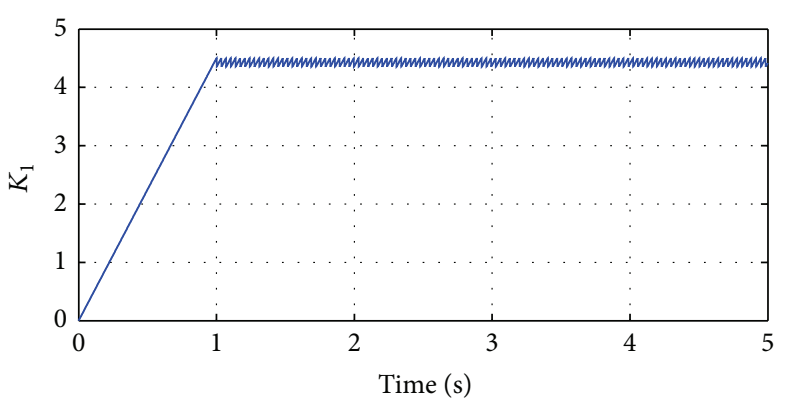

(a)

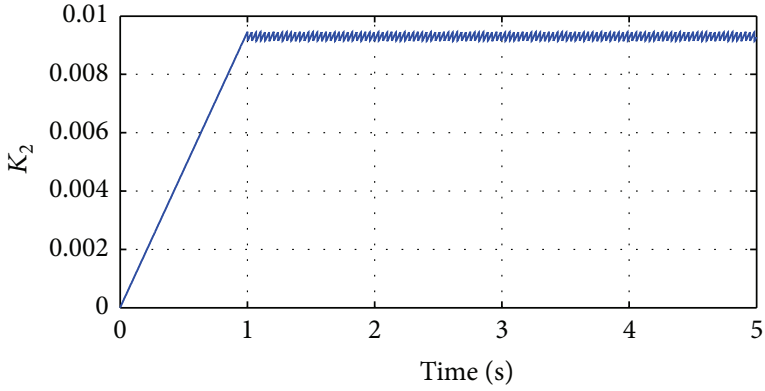

(b)

Figure 8: (a) Adaptive gain, $K_{1}$. (b) The dynamic gain $K_{2}$.

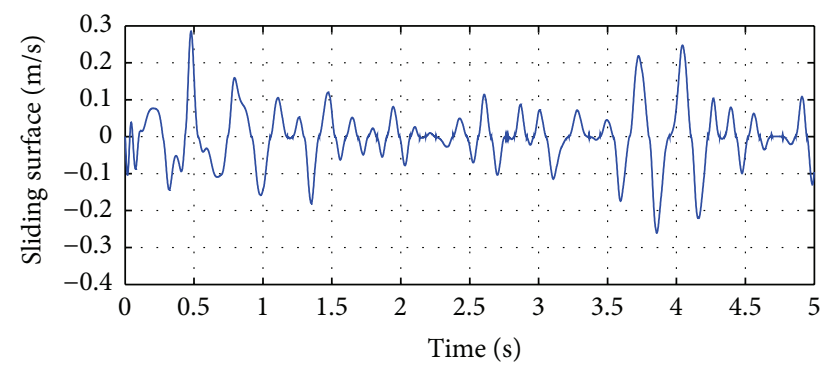

Figure 9: The sliding surface, $\sigma$.

To ensure that the provided control does not reduce the ride quality or lead to instability, the PSD of the unsprung mass acceleration should also be reduced and stay within definite limits. In Figure 12 the PSD of the unsprung mass acceleration has been shown.

For further analysis purposes the proposed controller scheme was applied to the suspension system under the effect of various classes of road profiles. The RMS values of the sprung mass acceleration, suspension deflection, and tire deflection for these cases under the influence of active and passive control are shown in Table 1. It can be deduced from the results shown in Table 1 that employing the proposed modified adaptive STA based controller improves the

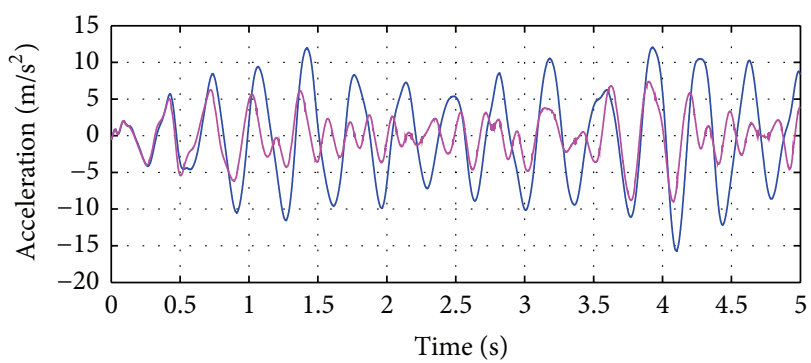

FIGURE 10: The sprung mass acceleration, $\ddot{z}_{s}$.

performance considerably. The proposed control strategy in this paper counters the effect of Lipschitz nonlinearities of the suspension system and the linearly growing perturbations represented by the road profile. Hence a controlled performance is provided enhancing the ride comfort while simultaneously maintaining road holding and constraining suspension stroke to specified mechanical limits.

\section{Conclusion}

In this work an active suspension control scheme was proposed based on the modified adaptive STA for a nonlinear suspension system faced with nonlinearities and road 
TABLE 1: RMS values of suspension deflection, tire deflection, and vertical acceleration.

\begin{tabular}{lcccccc}
\hline \multirow{2}{*}{ Road class } & \multicolumn{2}{c}{$z_{\text {su }}(\mathrm{m})$} & & $z_{\xi}(\mathrm{m})$ & & $\left(\ddot{z}_{s}\right)\left(\mathrm{m} / \mathrm{s}^{2}\right)$ \\
& Passive & Active & Passive & Active & Passive & Active \\
\hline B (good) & 0.000919 & 0.000272 & 0.000729 & 0.000682 & 0.5440 \\
C (average) & 0.0024 & 0.0019 & 0.0019 & 0.0013 & 1.4068 \\
D (poor) & 0.0051 & 0.0028 & 0.0040 & 0.0019 & 2.9747 \\
E (very poor) & 0.0103 & 0.0032 & 0.0081 & 0.0040 & 6.9462 \\
\hline
\end{tabular}

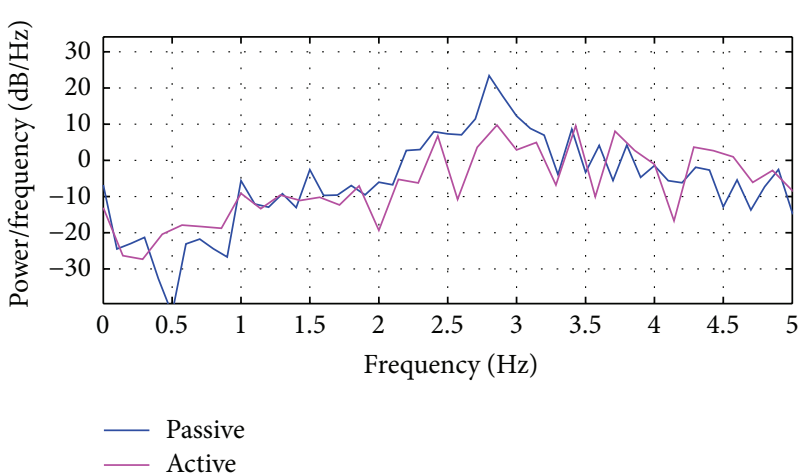

FIgURE 11: The PSD of sprung mass acceleration.

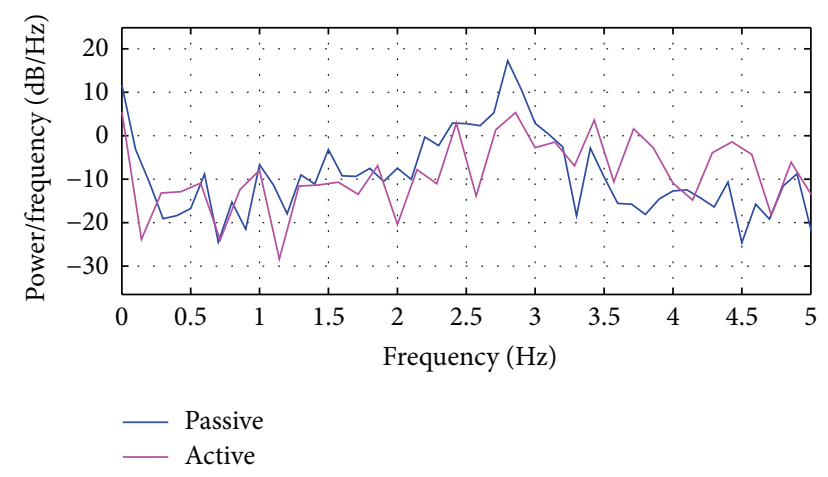

FIGURE 12: The PSD of unsprung mass acceleration.

excitations. The proposed control provided improvement in ride comfort for various classes of road profiles while ensuring that road holding capabilities of the vehicle were not compromised. In comparison to previous works in the literature the proposed controller was designed for linearly growing perturbations and was suitably employed for the suspension system. Simulation results were performed for different classes and presented results show the effectiveness of the proposed scheme.

\section{Conflict of Interests}

The authors declare that there is no conflict of interests regarding the publication of this paper.

\section{Acknowledgment}

This research was supported by the Basic Science Research Program through the National Research Foundation of Korea
(NRF) funded by the Ministry of Education, Science and Technology (Grant 2014R1A1A2A10056145).

\section{References}

[1] J. Rath, K. Veluvolu, and M. Defoort, "Simultaneous estimation of road profile and tyre road friction for automotive vehicle," IEEE Transactions on Vehicular Technology, p. 1, 2014.

[2] K. Tahboub, "Active nonlinear vehicle-suspension variable gain control," in Proceedings of the 13th IEEE International Mediterrean Symposium on Control and Automation: Intelligent Control, pp. 569-574, June 2005.

[3] Y. M. Sam, J. H. Osman, and M. R. Ghani, "A class of proportional-integral sliding mode control with application to active suspension system," Systems \& Control Letters, vol. 51, no. 3-4, pp. 217-223, 2004.

[4] N. Yagiz, Y. Hacioglu, and Y. Taskin, "Fuzzy sliding-mode control of active suspensions," IEEE Transactions on Industrial Electronics, vol. 55, no. 11, pp. 3883-3890, 2008.

[5] H. Li, H. Liu, H. Gao, and P. Shi, "Reliable fuzzy control for active suspension systems with actuator delay and fault," IEEE Transactions on Fuzzy Systems, vol. 20, no. 2, pp. 342-357, 2012.

[6] R. Rajamani and J. Hedrick, "Performance of active automotive suspensions with hydraulic actuators: theory and experiment," in Proceedings of the American Control Conference, vol. 2, pp. 1214-1218, IEEE, 1994.

[7] M.-M. Ma and H. Chen, "Disturbance attenuation control of active suspension with non-linear actuator dynamics," IET Control Theory \& Applications, vol. 5, no. 1, pp. 112-122, 2011.

[8] L. Xiao and Y. Zhu, "Sliding-mode output feedback control for active suspension with nonlinear actuator dynamics," Journal of Vibration and Control, 2013.

[9] K. C. Veluvolu, Y. C. Soh, W. Cao, and Z. Y. Liu, "Observer with multiple sliding modes for a class of nonlinear uncertain systems," in Proceedings of the American Control Conference (ACC '05), pp. 2445-2450, June 2005.

[10] K. C. Veluvolu and Y. C. Soh, "Multiple sliding mode observers and unknown input estimations for Lipschitz nonlinear systems," International Journal of Robust and Nonlinear Control, vol. 21, no. 11, pp. 1322-1340, 2011.

[11] K. C. Veluvolu and D. Lee, "Sliding mode high-gain observers for a class of uncertain nonlinear systems," Applied Mathematics Letters, vol. 24, no. 3, pp. 329-334, 2011.

[12] A. Chamseddine, H. Noura, and T. Raharijaona, "Control of linear full vehicle active suspension system using sliding mode techniques," in Proceedings of the IEEE International Conference on Control Applications (CCA '06), pp. 1306-1311, IEEE, Munich, Germany, October 2006.

[13] K. C. Veluvolu and Y. C. Soh, "Fault reconstruction and state estimation with sliding mode observers for Lipschitz non-linear 
systems," IET Control Theory \& Applications, vol. 5, no. 11, pp. 1255-1263, 2011.

[14] I. Boiko and L. Fridman, "Analysis of chattering in continuous sliding-mode controllers," IEEE Transactions on Automatic Control, vol. 50, no. 9, pp. 1442-1446, 2005.

[15] S. Kamal, A. Chalanga, J. A. Moreno, L. Fridman, and B. Bandyopadhyay, "Higher order super twisting algorithm," in Proceedings of the 13th International Workshop on Variable Structure Systems, pp. 1-5, 2014.

[16] M. Defoort, K. C. Veluvolu, J. J. Rath, and M. Djemai, "Adaptive sensor and actuator fault estimation for a class of uncertain Lipschitz nonlinear systems," International Journal of Adaptive Control and Signal Processing, 2015.

[17] H. Imine, L. M. Fridman, and T. Madani, "Steering control for rollover avoidance of heavy vehicles," IEEE Transactions on Vehicular Technology, vol. 61, no. 8, pp. 3499-3509, 2012.

[18] C. Evangelista, P. Puleston, and C. Kunusch, "Feasibility study of variable gain Super-Twisting control in fuel cells based systems," in Proceedings of the 13th International Workshop on Variable Structure Systems (VSS '14), pp. 1-6, IEEE, Nantes, France, July 2014.

[19] J. A. Moreno and M. Osorio, "Strict Lyapunov functions for the super-twisting algorithm," IEEE Transactions on Automatic Control, vol. 57, no. 4, pp. 1035-1040, 2012.

[20] J. A. Moreno, "On strict Lyapunov functions for some non-homogeneous super-twisting algorithms," Journal of the Franklin Institute, vol. 351, no. 4, pp. 1902-1919, 2014.

[21] J. A. Moreno, "Lyapunov analysis of non homogeneous SuperTwisting algorithms," in Proceedings of the 11th International Workshop on Variable Structure Systems (VSS '10), pp. 534-539, June 2010.

[22] V. I. Utkin, A. S. Poznyak, and P. Ordaz, "Adaptive super-twist control with minimal chattering effect," in Proceedings of the 50th IEEE Conference on Decision and Control and European Control Conference (CDC-ECC '11), pp. 7009-7014, December 2011.

[23] F. Plestan, Y. Shtessel, V. Brégeault, and A. Poznyak, "New methodologies for adaptive sliding mode control," International Journal of Control, vol. 83, no. 9, pp. 1907-1919, 2010.

[24] Y. Shtessel, M. Taleb, and F. Plestan, "A novel adaptive-gain supertwisting sliding mode controller: methodology and application," Automatica, vol. 48, no. 5, pp. 759-769, 2012.

[25] S. Toyama, F. Ikeda, and Y. Sorimachi, "A second order sliding mode controller for active suspension systems," in Proceedings of the International Conference on Control, Automation and Systems, pp. 18-23, October 2008.

[26] J. D. Sánchez-Torres, A. G. Loukianov, M. I. Galicia, J. Ruiz, and J. Rivera, "ABS and active suspension control via high order sliding modes and linear geometric methods for disturbance rejection," in Proceedings of the 8th International Conference on Electrical Engineering, Computing Science and Automatic Control, pp. 1-6, October 2011. 


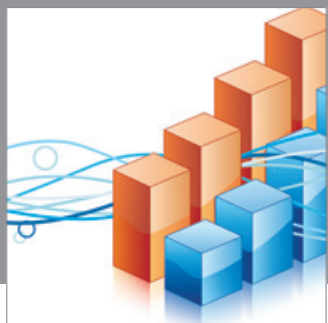

Advances in

Operations Research

mansans

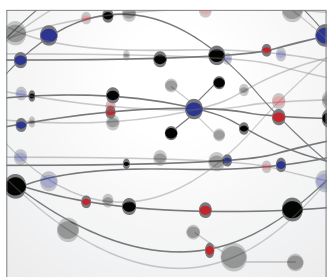

The Scientific World Journal
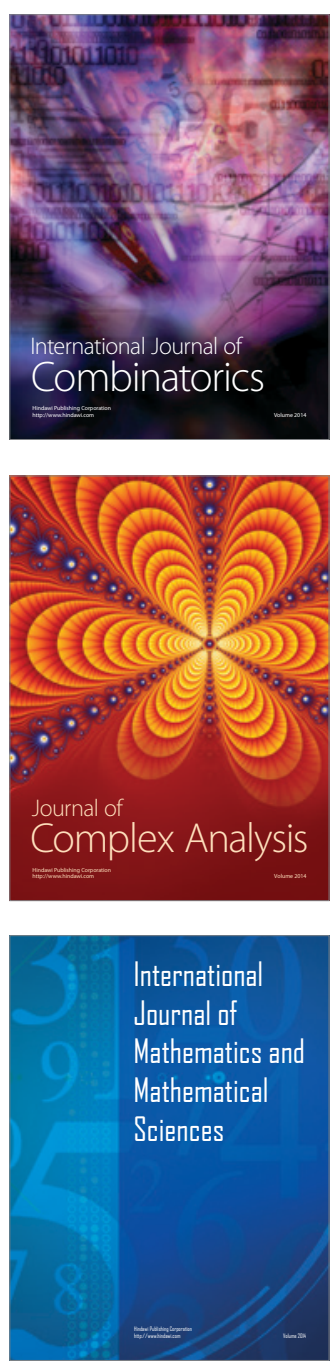
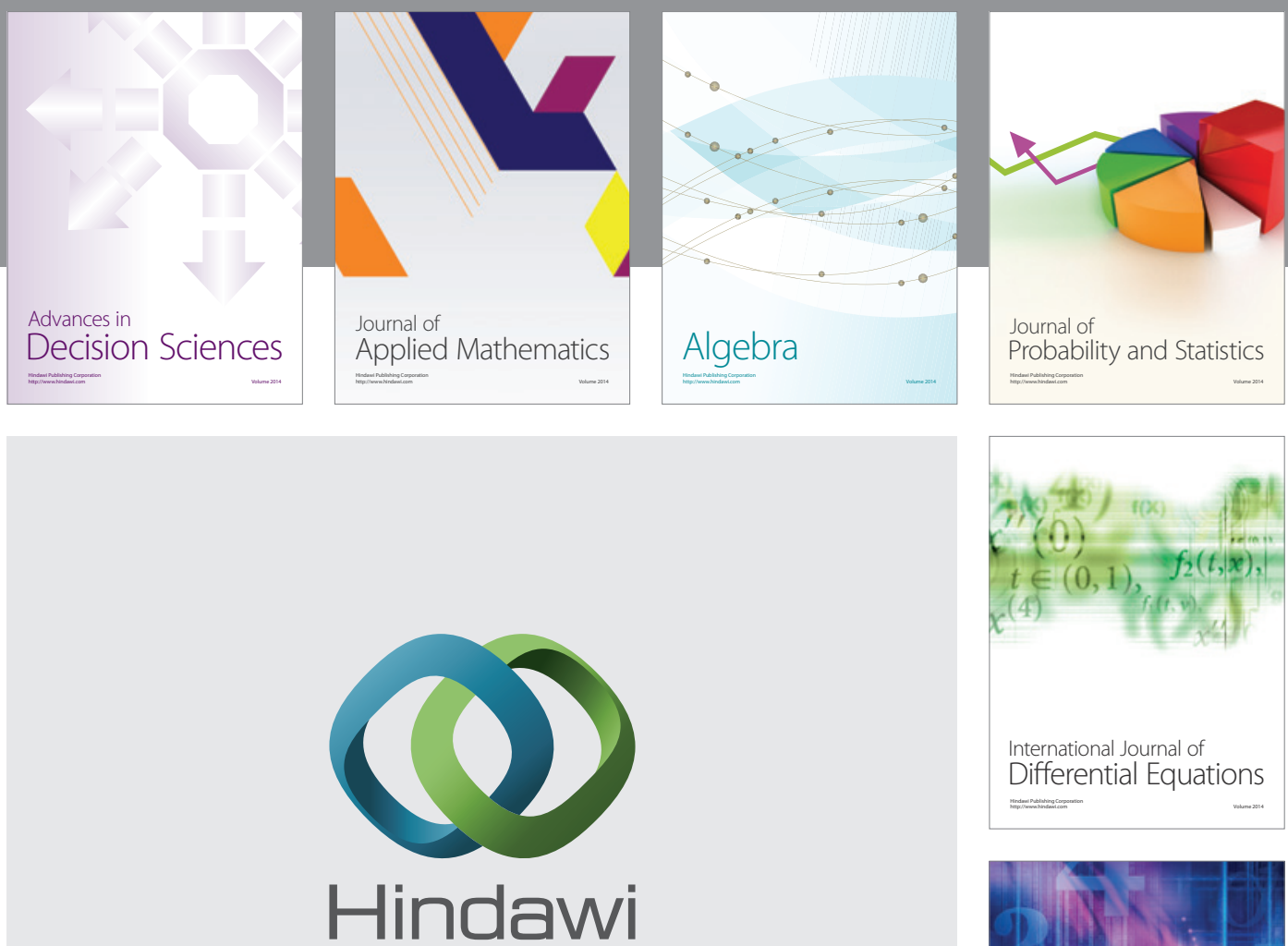

Submit your manuscripts at http://www.hindawi.com
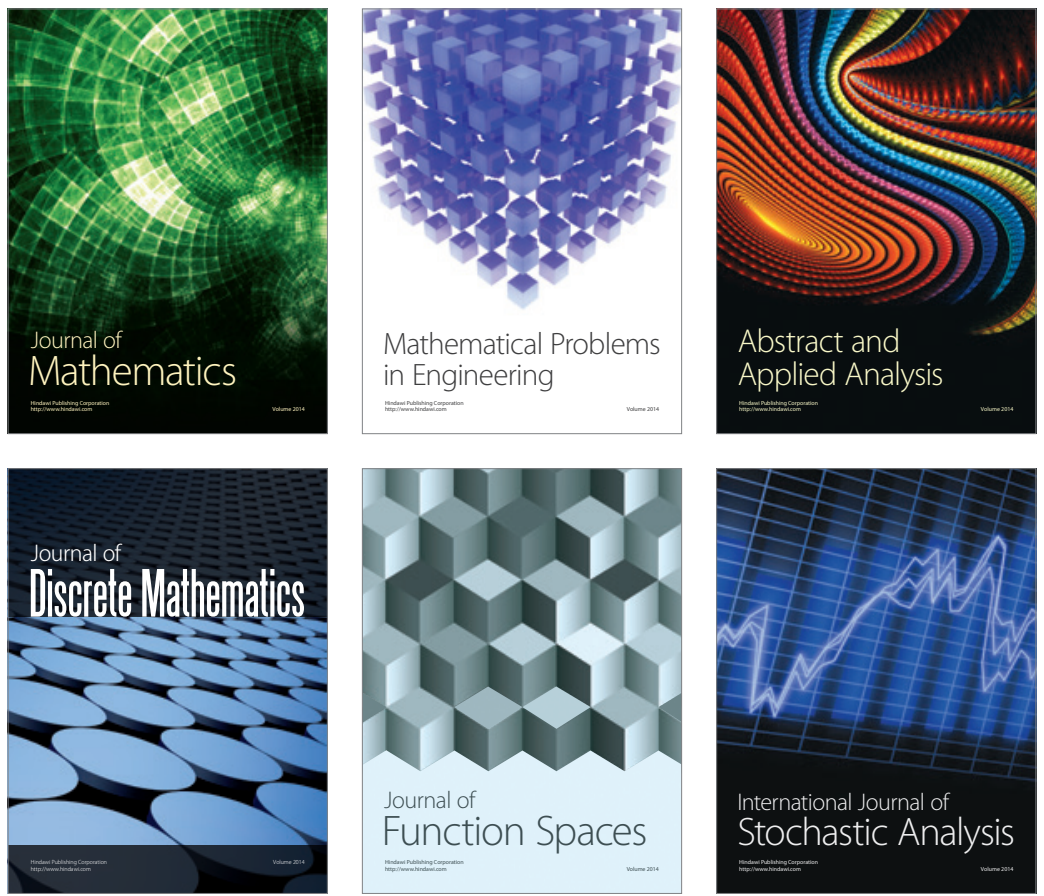

Journal of

Function Spaces

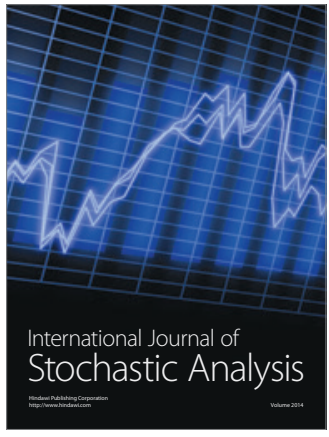

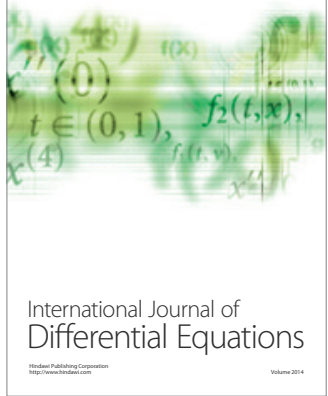
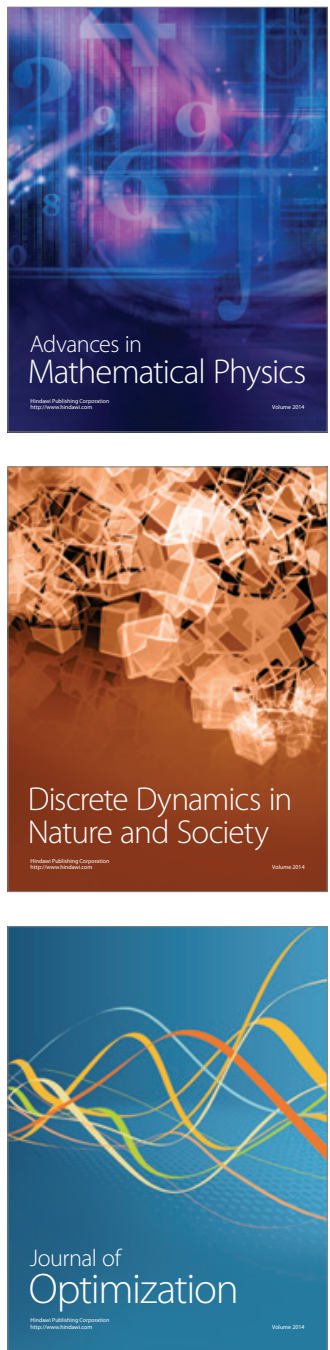